\title{
A note on the use of surface offset corrections in asteroseismic model fitting (Research Note)
}

\author{
Ian W. Roxburgh
}

\author{
Astronomy Unit, Queen Mary University of London, Mile End Road, London E1 4NS, UK \\ e-mail: I.W.Roxburgh@qmul .ac.uk
}

Received 24 March 2015 / Accepted 3 August 2015

ABSTRACT

\begin{abstract}
We critically investigate the practice of adding a power-law surface offset correction $f(v)$ to the frequencies of stellar models prior to seeking best fit models to an observed star. We show that surface layer independent indicators of the internal structure, phase shifts and separation ratios, are displaced in frequency by $f(v)$ and are therefore not the same as those of the model. Consequently such best fit models do not have exactly the same interior structure as the observed star. Using results on the star HD 177153 we show that the difference between observed and model frequencies for best fit models obtained using surface layer independent procedures have a wide range of different offsets which do not in general follow a Kjeldsen-like power law, and further that best fit models obtained using the offset correction procedure do not necessarily satisfy surface layer independent constraints on the internal structure.
\end{abstract}

Key words. stars: oscillations - asteroseismology - stars: interiors - methods: analytical - methods: numerical

\section{Introduction}

When seeking to find stellar models whose frequencies match those of an observed star many investigators use the so called surface frequency offset introduced by Kjeldsen et al. (2008) to compensate for our poor understanding of the structure of the outer layers of a star. Since the outer $1 \%$ by radius of a star contributes $\sim 15 \%$ to the acoustic radius $T$ (cf. Roxburgh 2014) a small error in modelling these layers can lead to large errors on the frequencies.

The reasoning behind the surface offset procedure is that, even for the Sun, the observed and "best" solar model frequencies differ by up to $15 \mu \mathrm{Hz}$, the offset approximately following a power law $f(v)=v^{\mathrm{mod}}-v^{\mathrm{obs}}=a\left(v^{\mathrm{obs}} / v_{0}\right)^{b}$ (where $v_{0}$ is any chosen reference frequency), so a similar offset should be expected for other stars. Using the solar Model S (Christensen-Dalsgaard et al. 1996), radial ( $\ell=0)$ frequencies from Lazrek et al. (1997), and a reference $v_{0}=3050 \mu \mathrm{Hz}$, Kjeldsen et al. fitted the offset with $a \sim 4.73 \mu \mathrm{Hz}, b \sim 4.90$.

In application to other stars the practice is to determine the exponent $b$ for the "best" solar model using the same set of physical assumptions as used to generate the model set to be searched for a best fit model to an observed star, and then determine the parameter $a$ from the average frequency and large separation of the model and observed star. This offset is then applied to each model prior to seeking a best fit model to a given observed frequency set. We remark that "best solar model" is subjective as there are many ways of defining "best": best fit to the frequencies, best fit to surface layer independent criteria, best fit to inferred properties such as the sound speed...

It is also the case that there is no empirical evidence to support using the scaled solar power law, nor does it rest on any theoretical basis; to the author it seems highly unlikely that the contribution of the outer layers of stars with different surface properties - mass, luminosity, radius, composition, mixing length parameter in convective modelling... can all be captured in a single parameter determined from the average frequency and large separation. As we show below the surface offsets of best fit models to HD 177153 determined by surface layer independent algorithms do not follow a Kjeldsen-like power law.

An important assumption behind using a surface offset correction is that the best fit model found by this procedure has the same interior structure as that of the observed star. We here show that this assumption is, in principle, incorrect. The addition of a function $f(v)$ to a model frequency set produces a displacement of $f(v)$ in the frequencies of signatures of the internal structure (phase shifts, separation ratios), which are then not the same for the model with and without the addition, and so the "corrected" frequencies do not reflect the interior structure of the model, but that of a different unknown model.

Further we show that using the Kjeldsen type offset one may find a best fit model from frequency matching whose interior structure is such that it does not satisfy surface independent model fitting criteria.

\section{Diagnostics of the stellar interior}

The frequencies $v_{n \ell}$ of a star or stellar model can always be represented in terms of "phase shifts" $\epsilon_{n \ell}$ as

$v_{n \ell}=\Delta\left[n+\ell / 2+\epsilon_{n \ell}\right], \quad \epsilon_{n \ell}=\frac{v_{n \ell}}{\Delta}-n-\ell / 2$,

for any convenient choice of a reference large separation $\Delta$. The $\epsilon_{n \ell}$ are determined by the structure of the star, both the interior and the outer layers, and for models can be determined by integrals over the structure. In the outer layers the contribution is essentially independent of angular degree $\ell$ whereas the contribution from the inner layers is $\ell$ dependent, so it is convenient to split the $\epsilon_{n \ell}$ into 2 components

$\epsilon_{n \ell} \equiv \epsilon_{\ell}\left(v_{n \ell}\right)=\alpha\left(v_{n \ell}\right)-\delta_{\ell}\left(v_{n \ell}\right)$ 


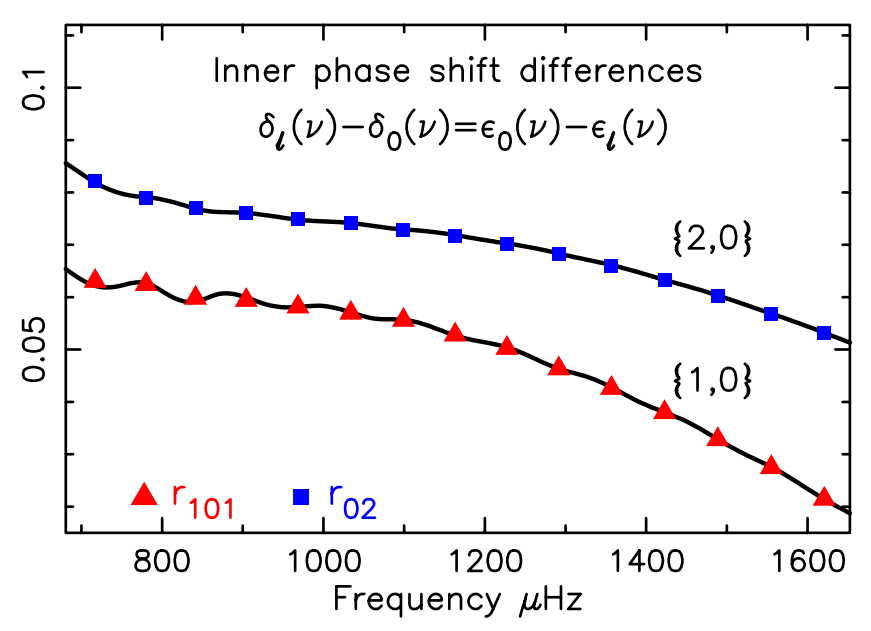

Fig. 1. Inner phase shift differences $\delta_{\ell}(v)-\delta_{0}(v)$ of a model of a $1.28 M_{\odot}$ and superimposed the interpolated curves for $\epsilon_{0}(v)-\epsilon_{\ell}(v)$. The points are the values of the separation ratios.

where the outer phase shift $\alpha(v)$ is an $\ell$ independent continuous function of $v$ determined by the structure of the outer layers, and the inner phase shifts $\delta_{\ell}(v)$ are $\ell$ dependent continuous functions of $v$ determined by the structure of the inner layers (cf. Roxburgh \& Vorontsov 1994, 2000). For a model these functions can be determined for any frequency - the eigenfrequencies being determined by continuity between the inner and outer solutions. Since the inner phase shifts $\delta_{\ell}(v)$ are determined by the inner structure alone these functions contain the information on the inner structure; different models having different phase shift functions $\delta_{\ell}(v)$.

The $\delta_{\ell}(v)$ are not directly observable, but their differences $\delta_{\ell}(v)-\delta_{0}(v)$ can be derived from the $\epsilon_{n \ell}$ by interpolation and subtraction. Interpolation in the discrete values $\epsilon_{\ell}\left(v_{n \ell}\right)$ to find the values at any frequency $v$ gives $\epsilon_{\ell}(v)=\alpha(v)-\delta_{\ell}(v)$ and subtracting the values for $\ell$ from those for $\ell=0$ eliminates $\alpha(v)$ giving the diagnostic

$\delta_{\ell}(v)-\delta_{0}(v)=\epsilon_{0}(v)-\epsilon_{\ell}(v)$.

The separation ratios $r_{01}, r_{02}, r_{101}$ (Roxburgh \& Vorontsov 2003, 2013) are another way of effecting this subtraction; if one uses linear interpolation to derive the value of $\epsilon_{1}(v)$ at the frequency $v_{n, 0}$ and subtracts this from $\epsilon_{n 0}=\epsilon_{0}\left(v_{n, 0}\right)$ one obtains the result

$r_{01}=\frac{v_{n, 0}-\left(v_{n, 1}+v_{n-1,1}\right) / 2}{v_{n, 1}-v_{n-1,1}}$

The much used 5 point separation $r_{101}$ is a higher order interpolation procedure which is more effective in eliminating the outer phase shift contribution $\alpha(v)$.

In Fig. 1 we the show the continuous inner phase shift differences derived from a model of mass $1.28 M_{\odot}$ and superposed the values from cubic spline interpolation in the $\epsilon_{n \ell}-$ the curves are indistinguishable. The points are the separation ratios which lie on these curves.

\section{The effect of adding a surface offset $f(v)$}

First we note that the inner phase shift differences $\delta_{\ell}(v)-\delta_{0}(v)$ are independent of the value assigned to $\Delta$. In terms of $\alpha, \delta_{\ell}$

$v_{n \ell}=\Delta\left[n+\ell / 2+\epsilon_{n \ell}\right]=\Delta\left[n+\ell / 2+\alpha\left(v_{n \ell}\right)-\delta_{\ell}\left(v_{n \ell}\right)\right]$ and expressing this in terms of a different $\Delta^{*}$ we have

$v_{n \ell}=\Delta^{*}\left[n+\ell / 2+\alpha\left(v_{n \ell}\right)-\delta_{\ell}\left(v_{n \ell}\right)\right]+v_{n \ell}\left[1-\frac{\Delta^{*}}{\Delta}\right]$.

Since $v_{n \ell}\left[1-\Delta^{*} / \Delta\right]$ is simply a linear function only of $v$, it can be absorbed into $\alpha(v)$; setting $\alpha^{*}(v)=\alpha(v)+v\left[1 / \Delta^{*}-1 / \Delta\right]$ gives

$v_{n \ell}=\Delta^{*}\left[n+\ell / 2+\epsilon_{n \ell}^{*}\right]=\Delta^{*}\left[n+\ell / 2+\alpha^{*}\left(v_{n \ell}\right)-\delta_{\ell}\left(v_{n \ell}\right)\right]$.

The inner phase shifts $\delta_{\ell}(v)$ as a function of $v$, which are determined solely by the inner structure of the star, are unchanged by this redefinition of $\alpha$, and are independent of the choice of $\Delta$.

Now let the actual frequencies of a model be $v_{n \ell}^{\mathrm{m}}$ (where the superscript $m$ indicates model) and the frequencies with offset $f(v)$ that match the observed frequencies $v_{n \ell}^{\mathrm{o}}$ be $v_{n \ell}^{\mathrm{m}}-f\left(v_{n \ell}^{\mathrm{o}}\right)$ then, for any value of $\Delta$,

$\epsilon_{\ell}^{\mathrm{o}}\left(v_{n \ell}^{\mathrm{o}}\right)=\frac{v_{n \ell}^{\mathrm{o}}}{\Delta}-n-\ell / 2, \quad \epsilon_{\ell}^{\mathrm{m}}\left(v_{n \ell}^{\mathrm{m}}\right)=\frac{v_{n \ell}^{\mathrm{m}}}{\Delta}-n-\ell / 2$.

Subtracting these equations gives

$\epsilon_{\ell}^{\mathrm{o}}\left(v_{n \ell}^{\mathrm{o}}\right)=\epsilon_{n \ell}^{\mathrm{m}}\left(v_{n \ell}^{\mathrm{m}}\right)-\frac{f\left(v_{n \ell}^{\mathrm{o}}\right)}{\Delta}$

where $f(v)$ is the offset. In terms of $\alpha$ and $\delta$ we have

$\alpha^{\mathrm{o}}\left(v_{n \ell}^{\mathrm{o}}\right)=\alpha^{\mathrm{m}}\left(v_{n \ell}^{\mathrm{m}}\right)-\frac{f\left(v_{n \ell}^{\mathrm{o}}\right)}{\Delta}, \quad \delta_{\ell}^{\mathrm{o}}\left(v_{n \ell}^{\mathrm{o}}\right)=\delta_{\ell}^{\mathrm{m}}\left(v_{n \ell}^{\mathrm{m}}\right)$

where since the term $f(v) / \Delta$ is a function only of $v$ it is added to the outer phase shift $\alpha^{\mathrm{m}}(v)$.

Hence for any frequency $v$ the $\delta$ differences (or equally the $\epsilon$ differences) satisfy

$\delta_{\ell}^{\mathrm{o}}(v)-\delta_{0}^{\mathrm{o}}(v)=\delta_{\ell}^{\mathrm{m}}(v+f(v))-\delta_{0}^{\mathrm{m}}(v+f(v))$.

The resulting differences $\delta_{\ell}^{\mathrm{o}}(v)-\delta_{0}^{\mathrm{o}}(v)$ of the observed frequencies are the same as those for the actual model frequencies but displaced in frequency by $f(v)$. Since it is the $\delta$ (or equivalently the $\epsilon$ ) differences as functions of frequency that are determined by the interior structure of a star it follows that, in principle, the observed and model star do not have the same interior structure. Whether or not this is important depends on the magnitude of the offset for the particular model compared with the errors in the $\delta$ differences which $\sim \sigma / \Delta$ where $\sigma$ is the error on the observed frequencies.

\section{Offset estimates from surface layer independent model fitting}

One way measuring frequency offsets is to compare the frequencies of best fit models to an observed star obtained using surface layer independent modelling techniques to those of the observed star. Results of such an analysis for the star HD 177153 using phase matching are reported in Roxburgh (2015). The star was observed by Kepler, and Silva-Aguirre et al. (2013) give 33 frequencies in the range $1600-2800 \mu \mathrm{Hz}, 11$ for each angular degree $\ell=0,1,2$, and a large separation $\Delta \sim 104 \mu \mathrm{Hz}$. Constraints on luminosity and radius were derived from bolometric flux and angular diameter measurement by Huber et al. (2012) and the HIPPARCos parallax (van Leeuwen 2007). Further details are given in Roxburgh (2015).

In Fig. 2 we show the offsets $f(v)=v^{\mathrm{m}}-v^{\mathrm{o}}$ for 2 of the 15 best fit models listed in that paper, $\chi_{\mathrm{ph}}^{2}$ being the $\chi^{2}$ of 


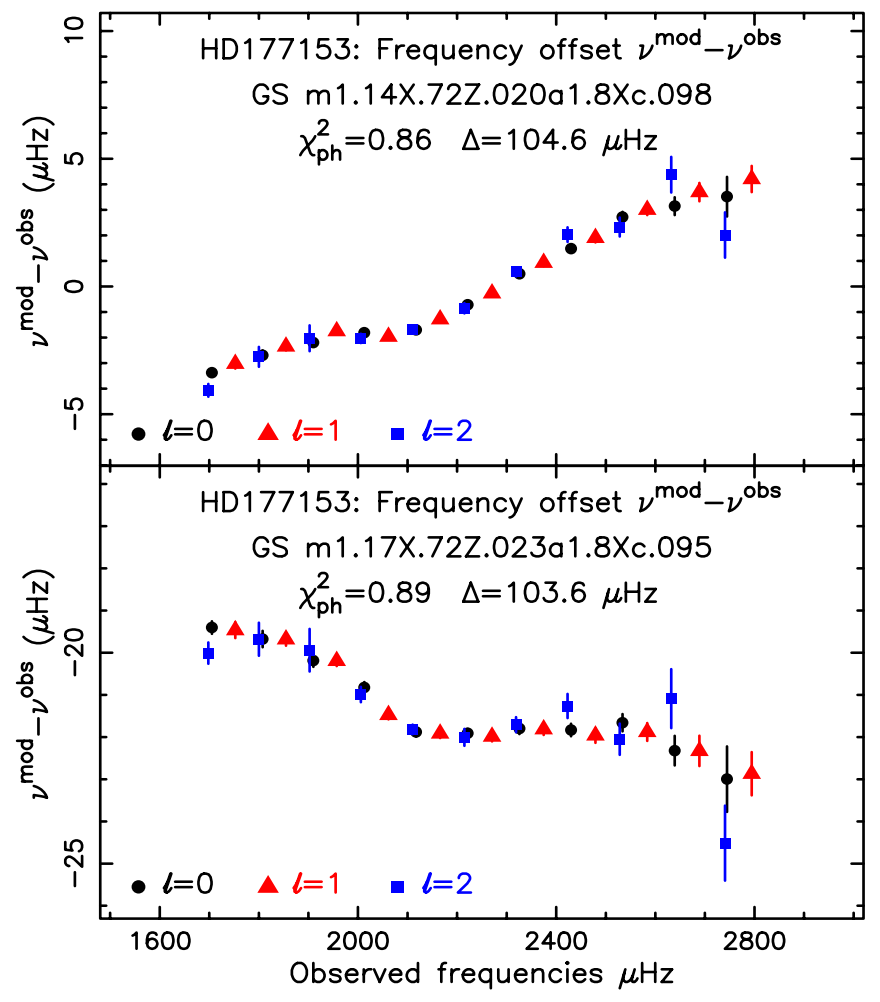

Fig. 2. Frequency offsets (model minus observed) for two of the best fit models to HD 177153 from surface layer independent model fitting. The offsets for these models are not power laws.

the phase match fit (Roxburgh 2015); the offsets do not have a Kjeldsen-like power law dependence. In general the offsets show a variety of functional forms: increasing with frequency, decreasing with frequency, some positive, some negative...

We then calculated the quality of the fits of the $N$ observed frequencies to the model values both without and with a Kjeldsen-like offset correction. The respective $\chi^{2}$ are

$\chi_{v}^{2}=\frac{1}{N} \sum_{n, \ell}\left(\frac{v_{n \ell}^{\mathrm{m}}-v_{n \ell}^{\mathrm{o}}}{\sigma_{n \ell}^{\mathrm{o}}}\right)^{2} \quad \chi_{\mathrm{K}}^{2}=\frac{1}{N} \sum_{n, \ell}\left(\frac{v_{n \ell}^{\mathrm{m}}-v_{n \ell}^{\mathrm{o}}-f\left(v_{n \ell}^{\mathrm{o}}\right)}{\sigma_{n \ell}^{\mathrm{o}}}\right)^{2}$.

The two model sets used in these analyses were relatively simple in that they did not include diffusion/radiative levitation so no attempt was made to fit the surface abundances (which were anyway poorly constrained). One set had GN93 relative abundances (Grevesse \& Noels 1993), the other GS98 (Grevesse \& Sauval 1998); the models were calculated using the OSCROX code (Roxburgh 2008). We followed the Kjeldsen offset correction procedure, normalising to a "best fit" solar model with relative abundances GN93 and GS98 that had the observed solar radius, solar luminosity and age (taken as $4.65 \times 10^{9} \mathrm{yr}$ ) and the lowest $\chi_{\mathrm{ph}}^{2}$ per degree of freedom in the surface independent matching. The results are shown in Fig. 3 using solar BiSON99 frequencies for modes of degree $\ell=0, n=14,25$ (Chaplin et al. 1999). The top panel shows the offset using the solar Model $\mathrm{S}$ (as in Kjeldsen et al. 2008) the bottom panel for our model set using GN93 abundances; the exponent $b=3.29$ for both the GN93 and GS98 solar models. In both diagrams we also give the weighted least squares power-law fit to all $\ell=0,1,2,3$ frequencies in the range $2000-4000 \mu \mathrm{Hz}$ where the exponent $b$ is somewhat larger that the Kjeldsen fit. We also give the $\chi^{2}$ of the fits.

We then applied the Kjeldsen offset to the frequencies of the 15 surface layer independent best fit models, the resulting $\chi_{\mathrm{K}}^{2}$ are

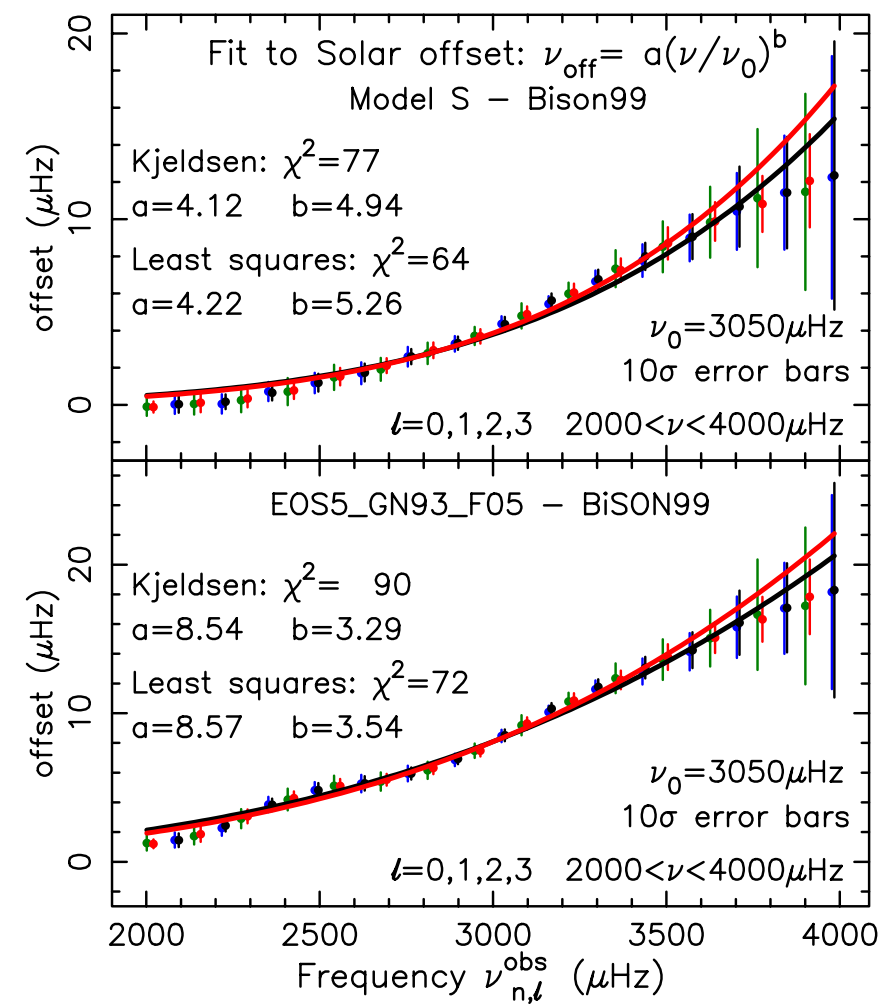

Fig. 3. Frequency offsets for the Sun and "best" solar models using BiSoN frequencies: top panel Model S, bottom panel reference solar model with GN93 abundances as used in model fitting. Black curve Kjeldsen et al. model, red curve weighted least squares fit.

Table 1. HD $177153 \chi^{2}$ for best phase shift models.

\begin{tabular}{lccccrr}
\hline \hline$Z_{i}$ & Mge9 & $\Delta$ & $\chi_{\mathrm{ph}}^{2}$ & $\chi_{v}^{2}$ & $\chi_{\mathbf{K}}^{\mathbf{2}}$ \\
\hline GS & m1.15X.72Z.021a1.8Xc.096 & 4.60 & 104.2 & 0.85 & 2740 & 8909 \\
GS & m1.16X.71Z.024a1.8Xc.095 & 4.45 & 104.5 & 0.96 & 128 & 2059 \\
GN & m1.16X.72Z.023a1.8Xc.096 & 4.57 & 104.4 & 0.92 & 604 & 3953 \\
GS & m1.16X.72Z.022a1.8Xc.095 & 4.57 & 103.9 & 0.85 & 7950 & 17023 \\
GS & m1.16X.72Z.023a1.8Xc.099 & 4.74 & 104.5 & 0.99 & 121 & 1984 \\
GS & m1.17X.72Z.023a1.8Xc.095 & 4.54 & 103.6 & 0.87 & 16260 & 28207 \\
GS & m1.17X.72Z.024a1.8Xc.097 & 4.70 & 104.1 & 1.00 & 2941 & 8266 \\
GN & m1.17X.72Z.024a1.8Xc.099 & 4.51 & 104.2 & 0.96 & 1904 & 6679 \\
GS & m1.18X.72Z.024a1.8Xc.099 & 4.48 & 103.4 & 0.89 & 22512 & 35852 \\
GS & m1.14X.72Z.020a1.8Xc.098 & 4.61 & 104.6 & 0.85 & 129 & 2359 \\
GS & m1.15X.71Z.023a1.8Xc.097 & 4.49 & 104.9 & 0.96 & 1768 & 6.6 \\
GS & m1.15X.72Z.022a1.8Xc.095 & 4.79 & 104.6 & 1.00 & 311 & 607 \\
GN & m1.15X.72Z.022a1.8Xc.095 & 4.61 & 104.7 & 0.91 & 275 & 785 \\
GS & m1.16X.74Z.019a1.8Xc.145 & 4.69 & 105.1 & 0.95 & 4313 & 530 \\
GS & m1.15X.71Z.022a1.8Xc0.097 & 4.31 & 104.4 & 1.04 & 630 & 4406 \\
\hline & & & & & & \\
\hline
\end{tabular}

Notes. Age9 is age in units of $10^{9} \mathrm{yr} \cdot \chi_{\mathrm{ph}}^{2}$ (phase shifts), $\chi_{v}^{2}$ (frequencies), $\chi_{\mathrm{K}}^{2}$ (Kjeldsen offset).

given in Table 1 along with the $\chi_{\mathrm{ph}}^{2}$ from phase matching and $\chi_{v}^{2}$ from direct frequency matching without the Kjeldsen offset correction. In all but 2 cases the fit with the Kjeldsen correction is considerably worse than that without, the notable exception being model GS: m1.15X.71Z.023a1.8Xc.097, where the Kjeldsen offset correction produces a much better fit. However this should not be taken as evidence that this model is better than the others; amongst the various $v^{\mathrm{m}}-v^{\mathrm{o}}$ frequency differences some model will have a difference that is more similar to the Kjeldsen offset 
Table 2. HD $177153 \chi^{2}$ for best Kjeldsen offset models.

\begin{tabular}{lcrrrrr}
\hline \hline$Z_{i}$ & Model & Age9 & $\Delta$ & $\chi_{\mathrm{ph}}^{2}$ & \multicolumn{1}{c}{$\chi_{v}^{2}$} & $\chi_{\mathbf{K}}^{\mathbf{2}}$ \\
\hline GN & m1.16X.74Z.022a1.8Xc.100 & 5.34 & 105.2 & 2.28 & 1466 & 6.4 \\
GS & m1.15X.71Z.023a1.8Xc.097 & 4.49 & 105.4 & 0.96 & 1768. & 6.6 \\
GN & m1.17X.73Z.022a1.6Xc.247 & 3.88 & 105.7 & 7.00 & 2797 & 16 \\
GN & m1.15X.71Z.023a1.8Xc.097 & 4.31 & 105.4 & 1.13 & 1559 & 17 \\
GS & m1.16X.70Z.024a1.6Xc.246 & 3.32 & 105.8 & 11.82 & 2958 & 27 \\
GN & m1.14X.72Z.024a2.0Xc.009 & 5.87 & 104.8 & 12.54 & 388 & 28 \\
GS & m1.19X.72Z.023a1.6Xc.296 & 3.20 & 105.8 & 15.33 & 2921 & 28 \\
GS & m1.17X.72Z.022a1.8Xc.146 & 4.14 & 105.5 & 1.92 & 22501 & 32 \\
GN & m1.18X.71Z.023a1.6Xc.297 & 2.88 & 105.9 & 19.16 & 3402 & 35 \\
GN & m1.18X.73Z.020a1.6Xc.299 & 3.16 & 106.0 & 15.75 & 4004 & 35 \\
GS & m1.17X.73Z.021a1.8Xc.145 & 4.41 & 105.4 & 1.26 & 1340 & 38 \\
GS & m1.19X.74Z.020a1.6Xc.297 & 3.48 & 105.8 & 12.58 & 3719. & 43 \\
GS & m1.18X.70Z.024a1.6Xc.297 & 2.79 & 105.8 & 20.52 & 2746 & 53 \\
GS & m1.16X.72Z.024a1.8Xc.096 & 4.93 & 105.3 & 1.36 & 1875 & 55 \\
GS & m1.16X.73Z.020a1.6Xc.247 & 3.88 & 105.9 & 7.24 & 4033 & 55 \\
\hline & & & & & & \\
\hline
\end{tabular}

Notes. $\chi_{\mathrm{ph}}^{2}$ (phase shifts), $\chi_{v}^{2}$ (frequencies), $\chi_{\mathrm{K}}^{2}$ (Kjeldsen offset).

than the others, and for this model the correction will improve the quality of the fit.

\section{Best fit models with Kjeldsen-like offset correction}

We then reversed the procedure searching for best fit models by frequency matching with the Kjeldsen offset correction. Table 2 gives the 15 models with the lowest $\chi_{\mathrm{K}}^{2}$ and the corresponding values of $\chi_{\mathrm{ph}}^{2}, \chi_{v}^{2}$. Whilst they have similar masses to the phase match best fit models in Table 1 they vary considerably in evolutionary stage with ages in the range between $2.79-5.87 \times 10^{9} \mathrm{yr}$, whereas the ages of the 15 best phase match models lie in the much narrower range of 4.31-4.74 $\times 10^{9} \mathrm{yr}$. More significantly most of these models do not satisfy surface layer independent model fitting criteria having a large $\chi_{\mathrm{ph}}^{2}$ and hence an interior structure that is not compatible with that of the observed star. The first model in Table 2, GN: m1.16X.74Z.022a1.8Xc.100, has the lowest $\chi_{\mathrm{K}}^{2}$ but a $\chi_{\mathrm{ph}}^{2}=2.28$ which for phase matching is very large - the probability that such a large $\chi^{2}$ is just a chance realisation of errors being less than $4 \times 10^{-4}$.

Model GS: m1.15X.71Z.023a1.8Xc.097 in Table 2 (which is also in Table 1) has both a low $\chi_{\mathrm{K}}^{2}$ and a $\chi_{\mathrm{ph}}^{2}=0.96$, thus satisfying the phase match criterion but, as remarked above, amongst the set of models that satisfy the phase match criterion some will have a frequency offset $v^{\mathrm{m}}-v^{\mathrm{o}}$ similar to that calculated using the Kjeldsen offset correction, whilst others do not. This model is one of those that does. In Fig. 4 we show the frequency differences $v^{\mathrm{m}}-v^{\mathrm{o}}$ of this model (the points) and the Kjeldsen offset values (the solid curve). Contrasting this with the offsets in Fig. 2 shows that this model just happens to have an offset $v^{\mathrm{m}}-v^{\mathrm{o}}$ that is close to the assumed offset correction.

\section{Conclusions}

We have shown that, in principle, the best fit model found by using the Kjeldsen et al. (2008) offset procedure does not have the same interior structure as the observed model one is seeking to fit since the phase shift differences (and separation ratios)

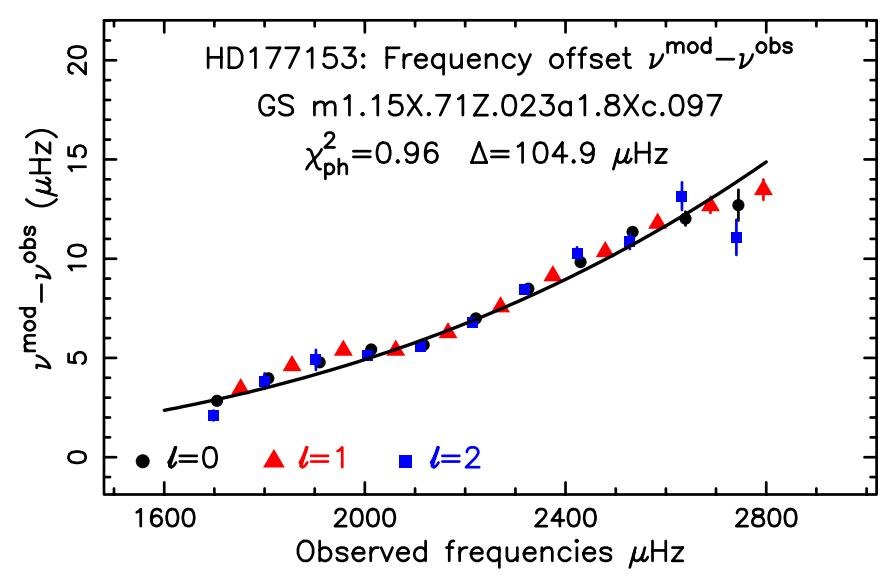

Fig. 4. Frequencies of model m1.15X.71Z.023a1.8Xc.097 minus observed values of HD 177153, the continuous curve is the offset calculated using the Kjeldsen algorithm.

are shifted in frequency by the value of the offset. This will only be important if the displacement in phase shift differences is comparable with, or greater than, the errors due to errors in the frequencies.

We then demonstrated that frequency offsets (model minus observed frequencies) of best fit models determined by surface layer independent fitting, phase or $\epsilon$ matching or separation ratios do not, in general, have a Kjeldsen-like power law character, and that best fit models using the Kjeldsen procedure do not, in general, satisfy surface layer independent model fitting constraints.

The inference from this study of just one star is that the use of surface offset à la Kjeldsen et al. (2008) has no justification. Were one to use this procedure it is essential to check that the resulting model also satisfies surface layer independent model fitting criteria - but one might just as well only use such surface layer independent modelling.

Acknowledgements. The author thanks a referee for comments that led to an improvement of the paper. He also gratefully acknowledges support from the Leverhulme Foundation under grant EM-2012-035/4 and from the UK Science and Technology Facilities Council (STFC) under grant ST/M000621/1.

\section{References}

Christensen-Dalsgaard, J., Dappen, W., Ajukov, S. V., et al. 1996, Science, 272, 1286

Chaplin, W. J., Elsworth, Y., Isaak, G. R., Miller, B. A., \& New, R. 1999, MNRAS, 308, 424

Grevesse, N., \& Noels, A. 1993, In Origin and Evolution of the Elements, eds. N. Prantzos, E. Vangioni-Flam, \& M. Casse (Cambridge Univ. Press), 15

Grevesse, N., \& Sauval, A. J. 1998, Space Sci. Rev., 85, 161

Huber, D., Ireland, M., Bedding, T., et al. 2012, ApJ, 760, 32

Kjeldsen, H., Bedding, T., \& Christensen-Dalsgaard, J. 2008, ApJ, 683, L175

Lazrek, M., Baudin, F., Bertello, L., et al. 1997, Sol. Phys, 175, 227

Roxburgh, I. W. 2008, Ap\&SS, 316, 75

Roxburgh, I. W. 2014, A\&A, 571, A88

Roxburgh, I. W. 2015, A\&A, 574, A45

Roxburgh, I. W., \& Vorontsov, S. V. 1994, MNRAS, 268, 880

Roxburgh, I. W., \& Vorontsov, S. V. 2000, MNRAS, 317, 141

Roxburgh, I. W., \& Vorontsov, S. V. 2003, A\&A, 411, 215

Roxburgh, I. W., \& Vorontsov, S. V. 2013, A\&A, 560, A2

Silva Aguirre, V., Basu, S., Brandao, I. M. et al. 2013, ApJ, 769, 141

van Leeuwen, F. 2007, A\&A, 474, 653 